Wie können die Steuerungsprobleme in regionalen Handlungssystemen bewältigt werden?

\title{
Innovationsaufgabe Nachhaltige Regionalentwicklung
}

\section{Die Arbeit an Konzepten Nachhaltiger Regionalentwicklung wird oft in Form von Projekten organisiert, die sich neben den etablierten Strukturen und Insti- tutionen bewegen. Die zahlreichen neven Ideen und Konzepte bleiben jedoch vielfach ohne Gestalfungsmacht und Bindungswirkung. Welche institutionellen und verfahrensbezogenen Innovationen sind notwendig, damit entsprechende Konzepte in die Praxis regionaler Politik Eingang finden?}

Von Rainer Lucas

m Rahmen der Agendaprozesse dominiert derzeit die lokale Ebene. Viele der dort angesprochenen Problemfelder, wie Verkehrsentwicklung, Wasserversorgung, Flächennutzung und Arbeitsmarktentwicklung, können jedoch nur auf regionaler Ebene adäquat bearbeitet werden.

Im föderalen Aufbau der Bundesrepublik Deutschland werden als Regionen Teilräume bezeichnet, die sich zwischen der Länderebene und der kommunalen Ebene bewegen. Diese werden vielfach durch übergeordnete Politikstrategien gebildet. Solch ein administrativer Weg ist im Rahmen einer Nachhaltigen Regionalentwicklung jedoch problematisch. Bereits die drei Gestaltungsdimensionen Soziales, Ökologie und Ökonomie weisen in der Regel unterschiedliche räumliche Bezugssysteme auf. So ist zum Beispiel der Naturraum nicht mit dem Arbeitsamtsbezirk identisch.

Vor diesem Hintergrund ist es methodisch von Bedeutung, den Analyse- und den Aktionsraum für Nachhaltige Entwicklung aus den Zielen und Problemstellungen zu begründen und an die unterschiedlichen räumlichen Strukturen, Potential- und Problemlagen anzupassen. Endogene Regionalentwicklung als ein Bezugspunkt der IÖW-Regionalforschung beruht darauf, diese Unterschiede herauszuarbeiten und an den spezifischen Potentialen für nachhaltige und innovative Regionalentwicklung anzusetzen. Auf dieser Basis kann im Laufe des Prozesses ein Gravitationszentrum für nachhaltiges Handeln entstehen, dessen regionale Grenzen entsprechend flexibel gehandhabt werden sollten.

Die Internationalisierung der Wirtschaftsbeziehungen, die Öffnung Osteuropas sowie der voranschreitende Prozeß der europäischen Inte- gration haben die Rahmenbedingungen für regionale Handlungssysteme in den letzten Jahren grundlegend gewandelt. Sie führen aus regionaler Sicht zu Steuerungsdefiziten, da (Welt-)Marktregulierung und supranationale Bürokratien kaum in der Lage sind, regionenspezifische und problemadäquate Lösungen für zukünftige Entwicklungen bereitzustellen. Die Kontextferne wird $\mathrm{zu}$ einem Steuerungsproblem.

\section{- Alte und neve Konzepte}

Nachhaltige Ansätze können an den Erfahrungen bestehender regionaler Konzepte und Strukturen anknüpfen. Wie die nachfolgende Tabelle 1 veranschaulicht, findet sich hier bereits vieles, was aktuell unter dem Stichwort Nachhaltigkeit in neuem Gewande daherkommt.

Konzeptionen Nachhaltiger Regionalentwicklung (NRE) sollten sich gegenüber herkömmlichen Ansätzen dadurch auszeichnen, daß sie bereits beim Problemzugang Wechselwirkungen zwischen sozialer, ökologischer und ökonomischer Dimension ermitteln und hieraus Konsequenzen für die Zielbildung sowie die Strukturierung der Handlungsfelder ableiten. Ein solches Vorgehen erhöht jedoch in erheblichem Maße die Zielund Programmkomplexität und damit die Anforderungen an das regionale Handlungssystem und einzelne Akteure.

Generell stehen für die gesellschaftliche Umsetzung der Nachhaltigkeit mehrere „Instrumentenkästen" zur Verfügung (1). Da Ordnungspolitik und auch ökonomische Anreize eher nationalstaatliche Aufgaben sind (Rahmensetzung), verbleiben fiur die Selbstregulation auf regionaler Ebene v.a. weiche Steuerungsinstrumente (Verhandlungsverfahren, Aufklärung). Die Ergebnisse dieser Prozesse sollten in den kommunalen und regionalen Planungsverfahren berïcksichtigt werden. Die Gestaltung derartiger Schnittstellen ist eine der großen Herausforderungen Nachhaltiger Regionalentwicklung.

Hinsichtlich ihres Entwicklungsverständnisses bewegen sich die Konzepte und Instrumente im Spannungsfeld exogener Anpassungsfähigkeit und dauerhafter (interner) Wandlungs- bzw. Innovationsfähigkeit (2). Auf die Bedeutung von effektiven Innovationssystemen für eine Nachhaltige Entwicklung verweist insbesondere die Enquete-Kommission des Deutschen Bundestages „Schutz des Menschen und der Umwelt“ (3). Die Notwendigkeit, die Innovationsfähigkeit zu stärken, ist weitgehend Konsens. Differenzen bestehen allerdings über die grundlegende Innovationsrichtung sowie die daraus abgeleiteten Innovationsschwerpunkte (4).

\begin{tabular}{|c|c|c|c|}
\hline Problemzugang & Zielsetzung & Handlungsfelder & Kooperationsmodi \\
\hline $\begin{array}{l}\text { Bedürnisse, } \\
\text { Märkte }\end{array}$ & $\begin{array}{l}\text { ökologische Optimierung } \\
\text { von Produkten } \\
\text { und Dienstleistungen, } \\
\text { Verhaltensänderungen }\end{array}$ & $\begin{array}{l}\text { Lendwirtschaft/ Ernährung, } \\
\text { ökologisches Baven/Wohnen, } \\
\text { regionale Dienstleistungs- } \\
\text { netzwerke }\end{array}$ & $\begin{array}{l}\text { Kooperation in der } \\
\text { Produktlinie, } \\
\text { Wertschöpfungsketten }\end{array}$ \\
\hline Infrastrukturen & $\begin{array}{l}\text { Optimierung von } \\
\text { Stoffströmen }\end{array}$ & $\begin{array}{l}\text { Abfollwirtschaft/ Recyding, } \\
\text { Wosserversorgung, } \\
\text { Verkehr, Flächennutzung }\end{array}$ & $\begin{array}{l}\text { Public-Private-Poit: } \\
\text { nerships, interkommunale } \\
\text { Kooperotion }\end{array}$ \\
\hline $\begin{array}{l}\text { Arbeit, } \\
\text { Einkommen }\end{array}$ & $\begin{array}{l}\text { Beschäftigungssicherung, } \\
\text { soziale Sicherung, } \\
\text { Qualifizierung, Selbsthilfe, } \\
\text { Chancengleichheit }\end{array}$ & $\begin{array}{l}\text { Arbeitsmarktpolitik, } \\
\text { Sozialpolitik, } \\
\text { Gleichstellungspolitik }\end{array}$ & $\begin{array}{l}\text { Arbeitsmarkikonferenz, } \\
\text { Arbeitslosenzentren, } \\
\text { Selbsthiffegruppen }\end{array}$ \\
\hline $\begin{array}{l}\text { Entwicklungsiaditeile } \\
\text { lëndicher Raume }\end{array}$ & $\begin{array}{l}\text { Aktivierung endogener } \\
\text { Potentiole, } \\
\text { Eigenstandigkeit }\end{array}$ & $\begin{array}{l}\text { Reglondpolifik, regionale } \\
\text { Strukiumolitik, } \\
\text { landwitschaft und } \\
\text { Touismus }\end{array}$ & $\begin{array}{l}\text { Abstimmung regionoler } \\
\text { Entwicklungs: } \\
\text { programme }\end{array}$ \\
\hline $\begin{array}{l}\text { Standortentwicklung, } \\
\text { Strukturwandel }\end{array}$ & $\begin{array}{l}\text { Verbesserung der } \\
\text { (ökologischen) } \\
\text { Wettbewerbs- und } \\
\text { Innovotionstöhigkeit }\end{array}$ & $\begin{array}{l}\text { Technologietransfer } \\
\text { technologieorientierte } \\
\text { Unternehmensgründungen }\end{array}$ & $\begin{array}{l}\text { Kooperation zwischen } \\
\text { Wirtschaftsförderung, } \\
\text { Technologiezentren } \\
\text { und Hochschulen }\end{array}$ \\
\hline
\end{tabular}

Quelle: eigene Darstellung 


\section{Soziale Innovationen no̊tig}

Welchen Beitrag können Konzepte Nachhaltiger Entwicklung zur regionalen Innovationsfähigkeit leisten? Provokative Antwort: Das wesentlich Neue sind nicht die Ziele und Inhalte, die sich mehr oder weniger in den regionalen Konzepten der letzten zehn Jahre bereits wiederfinden, sondern die prozeßbezogenen Gestaltungsanforderungen (vgl. zum Prozeß NRE den Beitrag von Molitor). Neu sind:

1. der programmatische Integrationsanspruch und die daraus resultierende Notwendigkeit, Fachpolitiken, Verwaltungsressorts und unterschiedliche Interessen aus den Bereichen Wirtschaft, Arbeit und Umwelt zu bündeln und

2. der prozeßbezogene Anspruch einer breiten Beteiligung regionaler Akteure in allen Phasen der Projektentwicklung.

Insofern geht es im wesentlichen um soziale Innovationen (5) als neuartige Verhaltensmuster von regionalen Akteuren, und um institutionelle Reformen (6). Die Zielsetzung dieses Wandels möchte ich mit drei Fragen umschreiben:

- Wie können institutionelle und prozeßbezogene Verfabren regional verankert werden, die sowohl die fachlichen als auch die akteursbezogenen Integrationsleistungen bewältigen?

- Wie kann eine Selbstbindung der beteiligten Akteure gestärkt werden, die dem Problemkontext der Nachhaltigkeit (sozial, ökologisch, ökonomisch) entspricht?

- Wie kann die Handlungsorientierung konkretisiert und operationalisiert werden?

Zum Erreichen dieser prozeßorientierten Ziele ist zusätzliches Engagement notwendig. Dieses wird sich nur einstellen, wenn regionale Nutzenpotentiale - wie z.B. Wettbewerbsvorteile, Marktchancen, Kostenreduktion und Imagebildung (7) - identifiziert und hieriber mit regionalen Akteuren kommuniziert wird.

\section{Innovationshemmnisse}

Die Schwächen Nachhaltiger Regionalentwicklung resultieren zum einen aus Faktoren, die in Problemen des gesamten Handlungssystems Region begründet sind. Konkurrenz zwischen einzelnen Kommunen, politische Handlungsblockaden, schwache intermediäre Organisationen und eine Regionalplanung ohne wirkliche Vollzugskraft schmälern die Durchsetzungschancen innovativer Lösungen. Insofern stellt sich unabhängig von den Nachhaltigkeitskonzepten die Frage, wie die institutionelle und organisatorische Entwicklungs- und Wandlungsfähigkeit von regionalen Handlungssystemen verbessert werden kann.

Zum anderen resultieren aus dem Dualismus alter und neuer Steuerungsformen zahlreiche Schnittstellenprobleme:

- Die Komplexität der Aufgabenstellung Nachhaltige Regionalentwicklung verleitet dazu, die Handlungsorientierung der Konzepte sehr breit anzulegen. $\mathrm{Zu}$ viele Themen und Handlungsfelder können jedoch zu einer Verzettelung der Kräfte führen. Eine erkennbare Prioritätensetzung hinsichtlich der zu bearbeitenden Probleme und Themen ist eine wichtige Erfolgsbedingung. Dies erhöht auch die Anschlußfähigkeit der Vorschläge für professionelle Akteure (u.a. Verbände, Verwaltungen, Unternehmen).

- Viele der institutionellen Innovationen (Runde Tische, Dialog-Foren, Netzwerke) sind außerhalb der etablierten regionalen Institutionen angesiedelt. Es besteht die Gefahr des Aufbaus von Nischenstrukturen jenseits der etablierten Entscheidungs- und Planungsvollzuige. Wenn normative und funktionale Regionalentwicklung getrennte Wege gehen, kann dies zu erheblichen Handlungsblockaden führen.

- Die regionalen Akteure haben in der Regel einen fach- und ressortbezogenen Hintergrund, der es ihnen schwer macht, mit einer integrativen Zielsetzung umzugehen. Diese Bindung an fachliche Eigenlogiken führt dazu, daß hinsichtlich der Nachhaltigkeitsdimensionen unterschiedliche Prioritäten gesetzt werden. Ist der fachliche Bezug oder die Berührung der eigenen Interessen nicht erkennbar, so stellt sich keine Selbstbindung der Akteure im Rahmen der regionalen Nachhaltigkeitskonzepte ein. Vor diesem Hintergrund ist es wichtig, fachübergreifende ,win-win-Strategien“ zu entwickeln.

\section{Komplexiłätsreduktion}

Insgesamt stellt sich die Aufgabe der Komplexitätsreduktion, um den Gegenstand einer regionalen Agenda handhabbar zu machen und an die konkreten Spielräume und Instrumente der Fachpolitiken heranzuführen (8). Von daher ist es notwendig, die Programmatik der Nachhaltigkeit auf der regionalen Ebene in ausgewählten Handlungsfeldern zu bündeln (z.B. raumbezogenes Stoffstrommanagement, verkehrssparende Siedlungsentwicklung, Regionalvermarktung landwirtschaftlicher Produkte). In diesen Feldern sind dann regionale Nutzenpotentiale einer
Verbesserung der regionalen Wertschöpfung, der Lebens- und Arbeitsbedingungen und der Umweltsituation zu erschließen. Schließlich ist es entscheidend, das Konzept in die vorhandenen Politikfelder zu integrieren.

Eine regionale Problemaufarbeitung und eine darauf aufbauende konzeptionelle Zielbildung sind also nicht hinreichend, um Nachhaltigkeit in regionalen Handlungssystemen zu verankern. Die Anforderungen ,integrierte Problemwahrnehmung“, ,Prioritätensetzung“, ,,Komplexitätsreduktion“ und „Beteiligung“ erfordern grundlegende soziale Innovationen und institutionelle Reformen. Diese müssen in den bestehenden regionalen Handlungssystemen durchgeführt werden, um eine dauerhafte Wirkung regionaler Nachhaltigkeitskonzepte zu erzielen.

\section{Anmerkungen}

(1) Renn, 0.: Ökologisch denken - sozial handeln: Die Realisierbarkeit einer nachhaltigen Entwicklung und die Rolle der Kultur- und Sozialwissenschaften. In: Kastenholz, H.G., G.H. Erdmann, M. Wolff (Hrsg.): Nachhaltige Entwicklung. Berlin, New York 1996, S. 79-117.

(2) Butzin, B. u.a.: Neue Strategien der Regionalentwicklung. Vergleich ausgewählter Fallstudien der Bundesrepublik, Bochum und München 1993.

(3) Zwischenbericht der Enquete-Kommission "Schutz des Menschen und der Umwelt - Ziele und Rahmenbedingungen einer nachhaltigen zukunftsverträglichen Entwicklung": Konzept Nachhaltigkeit, Deutscher Bundestag, Drucksache 13/7400, Bonn 1997, insb. S. 75.ff

(4) Gleich, Arnim von: Innovationsfähigkeit und Richtungssicherheit. Voraussetzungen für die ökologische Modernisierung der bundesrepublikanischen Stoffwirtschaft. In: Gleich, Arnim von, Simone Leinkauf, Stefan Zundel (Hrsg.): Surfen auf der Modernisierungswelle? Marburg 1997.

(5) Zapf, W. (Hrsg.): Theorie des sozialen Wandels, Köln 1989.

(6) IWÖ/IFOK: Institutionelle Reformen für eine Politik der Nachhaltigkeit. Berlin, New York 1998.

(7) Minsch, J. u.a.: Mut zum ökologischen Umbau. Innovationsstrategien für Unternehmen, Politik und Akteursnetze. Basel, Boston, Berlin 1996.

(8) Fürst, D.: Komplexitötsverarbeitung in der Planung (Stadt-, Regional- und Landesplanung) - am Beispiel der Regionalplanung. In: Archiv für Kommunalwissenschaften 1/1996, S. 20-37.

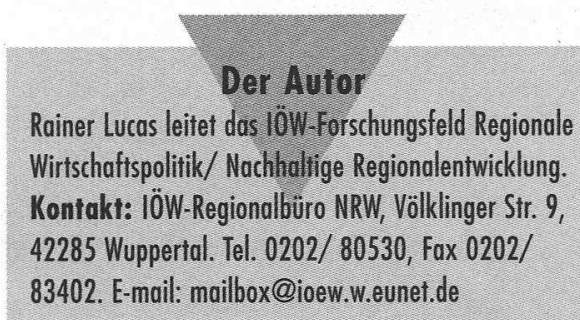


(c) 20I0 Authors; licensee IÖW and oekom verlag. This is an article distributed under the terms of the Creative Commons Attribution Non-Commercial No Derivates License (http://creativecommons.org/licenses/by-nc-nd/3.o/), which permits unrestricted use, distribution, and reproduction in any medium, provided the original work is properly cited. 